\title{
Aptamers and Aptamer-Coupled Biosensors to Detect Water-Borne Pathogens
}

\author{
Mariam Saad ${ }^{1,2 *}$ and Sebastien P. Faucher ${ }^{1,2}$ \\ ${ }^{1}$ Department of Natural Resources, Faculty of Agricultural and Environmental Sciences, McGill University, Montreal, QC, \\ Canada, ${ }^{2}$ Centre de Recherche en Infectiologie Porcine et Avicole (CRIPA), Université de Montréal, Faculté de Médecine \\ Vétérinaire, Saint-Hyacinthe, QC, Canada
}

OPEN ACCESS

Edited by:

Jingrang Lu,

United States Environmental Protection Agency, United States

Reviewed by:

Kai Wang,

Kent State University, United States William Thiel,

The University of lowa, United States

${ }^{*}$ Correspondence: Mariam Saad mariam.saad@mail.mcgill.ca

Specialty section:

This article was submitted to Frontiers in Microbiology, Microbiotechnology,

a section of the journal

Frontiers in Microbiology

Received: 18 December 2020 Accepted: 01 February 2021

Published: 19 February 2021

Citation:

Saad M and Faucher SP (2021) Aptamers and Aptamer-Coupled Biosensors to Detect

Water-Borne Pathogens.

Front. Microbiol. 12:643797. doi: 10.3389/fmicb.2021.643797
Aptamers can serve as efficient bioreceptors for the development of biosensing detection platforms. Aptamers are short DNA or RNA oligonucleotides that fold into specific structures, which enable them to selectively bind to target analytes. The method used to identify aptamers is Systematic Evolution of Ligands through Exponential Enrichment (SELEX). Target properties can have an impact on aptamer efficiencies. Therefore, characteristics of water-borne microbial targets must be carefully considered during SELEX for optimal aptamer development. Several aptamers have been described for key water-borne pathogens. Here, we provide an exhaustive overview of these aptamers and discuss important microbial aspects to consider when developing such aptamers.

Keywords: aptamer, SELEX, water-borne pathogens, viable but non-culturable, coliforms, aptasensors

\section{INTRODUCTION}

Access to water that is safe for use and consumption is a basic human right. As a result, most countries have strict guidelines, regulations and standards for managing water sources and water distribution systems to supply high quality water free from chemical and microbial contaminants. In most cases, microbial contaminants must be removed from the water before distribution. These microbes include pathogens that cause gastroenteritis, such as Cryptosporidium, Giardia, Norovirus, Rotavirus, Campylobacter, and E. coli (WHO, 2017). Other water-borne diseases are caused by pathogens growing inside water distribution systems or within engineered water systems, such as cooling tower, fountains, spas and humidifiers (Wang H. et al., 2017). The latter include Legionella pneumophila, Pseudomonas spp. and non-tuberculosis mycobacteria. In recent years, several studies have shown that a high proportion of water associated deaths and illnesses are due to the aforementioned three environmental water-borne pathogens (Gargano et al., 2017; Greco et al., 2020). In fact, L. pneumophila, the causative agent of Legionnaires disease, has become the number one cause of water-borne outbreaks in recent years (McClung et al., 2017). The presence of coliforms is not indicative of the presence of several key water-based pathogens that are of significance to public health (Payment and Locas, 2011). Consequently, specific detection methods are needed to ensure safe water from the source to the point of use. 
Monitoring and surveillance of specific water-borne microbes require robust detection methods. Challenges in select current detection methods for waterborne pathogens have been reviewed excellently in detail elsewhere (Ramírez-Castillo et al., 2015; Wang H. et al., 2017). In general, traditional microbial detection methods rely heavily on culture methods, which is fraught with several limitations. Culture methods are extremely time consuming and often require extensive material, specialized labor, and time. Culture recovery rates are also adversely affected by many factors such as the presence of competing microbes, the presence of viable but non-culturable (VBNC) cells, methods used for concentration of the sample or enrichment of the target microbe and sample type (bulk water or biofilm) (Wang H. et al., 2017). Drawbacks with culture techniques has led to a shift toward the use of molecular methods, including PCR, quantitative PCR (qPCR), high throughput sequencing, and immunoassays such as ELISA, immunochromatography and immuno-lateral flow assays. The most widely used molecular method is qPCR (Ramírez-Castillo et al., 2015; Wang H. et al., 2017). The advantage of qPCR, over conventional culture techniques, is more rapid turn-around times, high sensitivities and specificities, lower limits of detection, as well as an ability to detect VBNC cells. However, by detecting live, VBNC and dead cells qPCR leads to an overestimation of microbial burden. Additionally, qPCR involves multiple sample processing steps which requires specialized labor. $\mathrm{qPCR}$ is also inhibited by several compounds routinely found in water samples resulting in possible false negatives (Gentry-Shields et al., 2013).

Biosensors can mitigate some of the problems associated with traditional detection methods (Ahmed et al., 2014). They are analytical devices used to quantify or detect a specific analyte (Turner, 2013). Qualities of biosensors includes high specificity, high sensitivity, multiplexing capability, costeffectiveness, portability and ease of use (Ahmed et al., 2014; Kumar et al., 2018; Cesewski and Johnson, 2020; McConnell et al., 2020). A biosensor set-up typically consists of three elements. A biorecognition element, which upon interaction with a target, produces a physico-chemical signal that is converted by a transducing element into a signal captured by a detection element (Turner, 2013). Biosensors are categorized based on either their transducing element (mechanical, optical, electrochemical) or the nature of the biorecognition element (affinity, catalytic) (Ahmed et al., 2014).

A versatile and stable biorecognition element is a critical component of any biosensing platform (Ahmed et al., 2014; Kumar et al., 2018). Antibodies are the most used bioreceptors in biosensor development and research, but aptamers are an increasingly widespread popular alternative (Song et al., 2008; Morales and Halpern, 2018). Aptamers are single stranded DNA or RNA oligonucleotides that fold into specific complex structures and interact with their targets via shape complementarity, hydrogen bonding, electrostatic interactions and stacking interactions (McKeague et al., 2015). Besides having high affinities and selectivity, they can bind to a wide range of targets from small non-immunogenic compounds to whole cells (McKeague et al., 2015). Aptamers can be generated in vitro in conditions one can preferentially select making them stable and versatile for a variety of applications (Song et al., 2008). They are cost-effective to synthesize with minimal batch to batch variation (Strehlitz et al., 2012; McConnell et al., 2020). Their easily modifiable nature facilitates functionalization on sensing surfaces (Song et al., 2008; McConnell et al., 2020). Their inherent small size also promotes high packing densities during functionalization (Song et al., 2008; Crivianu-Gaita and Thompson, 2016). In this minireview, we will briefly provide examples of aptamers with potential for detection of waterborne pathogens and discuss microbial determinants for the development of optimal aptamers and thus improved aptamercoupled biosensors. Examples of aptamers is provided in Table 1 and a complete list of aptasensing platforms is provided in Supplementary Table 1 .

\section{APTAMER DEVELOPMENT}

Aptamers are typically identified by SELEX (Systematic Evolution of Ligands through Exponential Enrichment). SELEX is an iterative process where repeated exposure of a target to a large pool of random oligonucleotides results in the gradual enrichment of specific sequences that bind with the highest affinity to the target. Since the technique's inception in 1990, many variations of the original SELEX method have been published (Darmostuk et al., 2015). These experimental variations differ based on desired aptamer properties and details have been reviewed elsewhere (Wang et al., 2019). Of note, cell-SELEX can be used to select aptamers against whole cells in solution, to ensure cell surface target epitopes are in their native state (Kaur, 2018). This method is particularly useful for developing aptamers to detect water-borne pathogens. CellSELEX may include counter-selection steps to remove sequences binding to non-target microbes thus minimizing cross-reactivity and improving the specificity of the resulting aptamers (see Table 1 for examples).

Several aptamer-coupled biosensing systems or aptasensors have been described for the detection of water-borne pathogens or toxins accumulating in water (Table 1 and Supplementary Table S1) with the majority targeting bacterial pathogens. Nevertheless, none have been officially adopted for routine detection of water-borne pathogens. The development of successful aptamer-coupled biosensors to detect water-borne pathogens requires a multi-pronged approach. Besides intricate knowledge of the sensing system, its transducer, the physicochemical phenomenon that mediate signal responses, and a deep understanding of aptamer chemistries, careful consideration of the physiology and ecology of the target microorganism is required. This is because physio-ecological factors affect microbial morphologies and surface structures and thus the presence of aptamer targets (Figure 1). Although several works discuss transducing systems and aptamer design and chemistries in detail, relatively fewer studies consider the physioecological context of water-borne microbes for sensing platforms. Since most aptamers and aptasensing systems described in the literature detects water-borne bacterial pathogens, properties of bacteria are discussed in more detail to illustrate the importance 
TABLE 1 | Aptamers developed against water-borne bacteria.

\begin{tabular}{|c|c|c|c|c|c|c|c|}
\hline Aptamer name & Target & $\begin{array}{l}\text { Culture } \\
\text { condition }^{a}\end{array}$ & $\begin{array}{l}\text { OD/Growth } \\
\text { stage }^{a, b}\end{array}$ & Counter-Selex Strains $^{c}$ & Type of sensors & LOD & References \\
\hline \multicolumn{8}{|l|}{ Norovirus } \\
\hline \multirow[t]{2}{*}{ AG3 } & MuNoV & NA & NA & Feline calicivirus (FCV) & Electrochemical & $\begin{array}{l}180 \text { virus } \\
\text { particles }\end{array}$ & Giamberardino et al., 2013 \\
\hline & & & & NA & Optical (colorimetric) & 200 virus/ml & Weerathunge et al., 2019 \\
\hline \multirow[t]{2}{*}{$\begin{array}{l}\text { Aptamer } \\
\text { 25/SMV-25 }\end{array}$} & SMV & $N A$ & $N A$ & $\begin{array}{l}\text { HuNoV-negative human stool } \\
\text { suspension, bead-antibody } \\
\text { complex }\end{array}$ & $N A$ & $N A$ & Escudero-Abarca et al., 2014 \\
\hline & $\begin{array}{l}\text { Non-toxic norovirus Gll } \\
\text { capsid recombinant }\end{array}$ & & & $N A$ & $\begin{array}{c}\text { Optical } \\
\text { (Chemiluminescence) }\end{array}$ & $80 \mathrm{ng} / \mathrm{ml}$ & Kim B. et al., 2018 \\
\hline \multirow[t]{2}{*}{$\begin{array}{l}\text { Aptamer } \\
21 / \text { SMV-21 }\end{array}$} & SMV & $N A$ & $N A$ & $\begin{array}{l}\text { HuNoV-negative human stool } \\
\text { suspension, bead-antibody } \\
\text { complex }\end{array}$ & $N A$ & $N A$ & Escudero-Abarca et al., 2014 \\
\hline & $\begin{array}{l}\text { Norovirus Group II } \\
\text { (recombinant VLP) }\end{array}$ & & & $N A$ & Electrochemical & $100 \mathrm{pM}$ & Chand and Neethirajan, 2017 \\
\hline \multicolumn{8}{|l|}{ C. parvum } \\
\hline \multirow[t]{2}{*}{ R4-6 } & Oocysts & $N A$ & $N A$ & Giardia duodenalis cysts & Electrochemical & 100 oocysts & lqbal et al., 2015 \\
\hline & & & & NA & Electrochemical & 50 oocysts & lqbal et al., 2019 \\
\hline Min_Crypto2 & Oocysts & $N A$ & $N A$ & $N A$ & Optical fluorescence & 5 oocysts & Hassan et al., 2021 \\
\hline \multicolumn{8}{|l|}{ Acinetobacter } \\
\hline \multirow[t]{3}{*}{ Aci49 } & $\begin{array}{l}\text { Whole-cell-A. baumannii } \\
\text { (ATCC 19606) }\end{array}$ & $\begin{array}{c}\text { BHI broth, } \\
37^{\circ} \mathrm{C} \text {, overnight }\end{array}$ & $0.4 / E$ & $\begin{array}{l}\text { Acinetobacter Iwoffii, Acinetobacter } \\
\text { calcoaceticus, and } 11 \text { species }\end{array}$ & Optical (colorimetric) & $10^{3} \mathrm{CFU} / \mathrm{ml}$ & Rasoulinejad and Gargari, 2016 \\
\hline & & & & $N A$ & Optical (fluorescence) & $3 \mathrm{CFU} / \mathrm{ml}$ & Li et al., 2020 \\
\hline & & & & NA & Optical (fluorescent) & $10 \mathrm{CFU} / \mathrm{ml}$ & Yang et al., 2020 \\
\hline \multirow[t]{4}{*}{ AB aptamer } & Whole-cell A. baumannii & NR & NR & NR & Optical (colorimetric) & $450 \mathrm{CFU/r \times n}$ & Wu et al., 2018 \\
\hline & & & & NA & Optical (fluorescence) & $10^{5} \mathrm{CFU} / \mathrm{ml}$ & Su et al., 2020 \\
\hline & & & & $N A$ & Optical (fluorescence) & $100 \mathrm{CFU} / \mathrm{ml}$ & Su et al., 2020 \\
\hline & & & & $N A$ & Optical (fluorescence) & $300 \mathrm{CFU} / \mathrm{ml}$ & Bahari et al., 2021 \\
\hline \multicolumn{8}{|l|}{ Aeromonas } \\
\hline Apt1 & Whole-cell (A. hydrophila) & $\mathrm{LB}, 37^{\circ} \mathrm{C}, 18 \mathrm{~h}$ & NR & NR & Optical (Fluorescence) & $1.5 \mathrm{CFU} / \mathrm{ml}$ & Zhu et al., 2019 \\
\hline \multicolumn{8}{|l|}{ Campylobacter } \\
\hline \multirow{2}{*}{$\begin{array}{l}\text { Aptamer C2 and } \\
\text { Aptamer C3 }\end{array}$} & Surface protein (C. jejuni) & NR & NR & NR & Optical (fluorescence) & $2.5 \mathrm{CFU} / \mathrm{ml}$ & Bruno et al., 2009 \\
\hline & & & & $N A$ & Optical (colorimetric) & 5-10 CFU/ml & Bruno and Sivils, 2017 \\
\hline \multirow[t]{3}{*}{ ONS-23 } & Whole-cell (C. jejuni A9a) & $\begin{array}{c}\mathrm{BBL} \text { brucella } \\
\text { broth, } 42^{\circ} \mathrm{C} \text {, } \\
48 \mathrm{~h} \text {, } \\
\text { microaerophillic } \\
\text { conditions }\end{array}$ & $\mathrm{PE}^{*}$ & $\begin{array}{c}20 \text { strains (enteric, non-enteric, } \\
\text { lactic acid) }\end{array}$ & $N A$ & $N A$ & Dwivedi et al., 2010 \\
\hline & & & & $N A$ & Optical (colorimetric) & $10 \mathrm{CFU} / \mathrm{ml}$ & Dehghani et al., 2018 \\
\hline & & & & NA & Optical (colorimetric) & $7.2 \times 10^{5} \mathrm{CFU} / \mathrm{ml}$ & Kim Y. J. et al., 2018 \\
\hline CJA1 & Whole-cell (C. jejuni) & & & NR & Optical (colorimetric) & $10 \mathrm{CFU} / \mathrm{ml}$ & Chen et al., 2020 \\
\hline
\end{tabular}


TABLE 1 | Continued

\begin{tabular}{|c|c|c|c|c|c|c|c|}
\hline Aptamer name & Target & $\begin{array}{l}\text { Culture } \\
\text { condition }^{a}\end{array}$ & $\begin{array}{l}\text { OD/Growth } \\
\text { stage }^{a, b}\end{array}$ & Counter-Selex Strains $^{c}$ & Type of sensors & LOD & References \\
\hline \multicolumn{8}{|l|}{ Cyanobacteria } \\
\hline ATX8 & Anatoxin-a (ATX) & $N A$ & $N A$ & ATX free beads & Electrochemical & $0.5 \mathrm{nM}$ & Elshafey et al., 2015 \\
\hline \multirow{2}{*}{$\begin{array}{l}\text { MC-LR } \\
\text { aptamer/AN6 }\end{array}$} & Microcystin-LR & $N A$ & $N A$ & Blank sepharose beads & Electrochemical & $10 \mathrm{pM}$ & Ng et al., 2012 \\
\hline & & & & $N A$ & Optical (fluorescence) & $0.002 \mathrm{ng} / \mathrm{ml}$ & Lv et al., 2017 \\
\hline \multicolumn{8}{|l|}{ E. coli } \\
\hline \multirow[t]{2}{*}{ L9F } & $\begin{array}{l}\text { O111-LPS (E. coli } \\
\text { O111:K58) }\end{array}$ & $\begin{array}{l}35^{\circ} \mathrm{C}, \mathrm{TSB} \\
\text { overnight }\end{array}$ & NR & NR & $N A$ & $N A$ & Bruno et al., 2008 \\
\hline & & & & $N A$ & Electrochemical & $112 \mathrm{CFU} / \mathrm{ml}$ & Luo et al., 2012 \\
\hline \multirow[t]{2}{*}{ Eco4R/ECAll } & $\begin{array}{l}\text { Outer membrane protein } \\
(\mathrm{OMP})-E \text {. coli } 8739\end{array}$ & $\begin{array}{l}37^{\circ} \mathrm{C} \text {, blood } \\
\text { agar, overnight }\end{array}$ & NR & NR & $N A$ & NA & Bruno et al., 2010 \\
\hline & & & & NA & Electrochemical & NR & Queirós et al., 2013 \\
\hline \multirow[t]{2}{*}{ Eco4F } & OMP-E. coli 8739 & $\begin{array}{l}37^{\circ} \mathrm{C} \text {, blood } \\
\text { agar, overnight }\end{array}$ & NR & NR & $N A$ & $N A$ & Bruno et al., 2010 \\
\hline & & & & $N A$ & $\begin{array}{c}\text { Optical } \\
\text { (colorimetric/fluorescence) }\end{array}$ & $300 \mathrm{CFU} / \mathrm{ml}$ & Bruno, 2014 \\
\hline \multirow[t]{4}{*}{ Eco3R/ECAl } & OMP-E. coli 8739 & $\begin{array}{l}37^{\circ} \mathrm{C} \text {, blood } \\
\text { agar, overnight }\end{array}$ & NR & NR & $N A$ & $N A$ & Bruno et al., 2010 \\
\hline & & & & $N A$ & Electrochemical & NR & Queirós et al., 2013 \\
\hline & & & & $N A$ & $\begin{array}{c}\text { Optical } \\
\text { (colorimetric/fluorescence) }\end{array}$ & $300 \mathrm{CFU} / \mathrm{ml}$ & Bruno, 2014 \\
\hline & & & & NA & $\begin{array}{l}\text { Optical (Evanescent wave } \\
\text { fiber optics) }\end{array}$ & $0.1 \mathrm{nM}$ & Queirós et al., 2014 \\
\hline E1 & $\begin{array}{l}\text { Whole cell (E. coli fecal } \\
\text { isolate) }\end{array}$ & $\mathrm{NB}, 37^{\circ} \mathrm{C}$ & $0.45 / \mathrm{E}$ & $\begin{array}{l}\text { E. coli (non-fecal isolate), other } \\
\text { fecal isolates }\end{array}$ & $N A$ & $N A$ & Kim et al., 2013 \\
\hline \multirow[t]{3}{*}{ E2 } & $\begin{array}{l}\text { Whole cell (E. coli fecal } \\
\text { isolate) }\end{array}$ & $\mathrm{NB}, 37^{\circ} \mathrm{C}$ & $0.45 / \mathrm{E}$ & $\begin{array}{l}\text { E. coli (non-fecal isolate), other } \\
\text { fecal isolates }\end{array}$ & $N A$ & $N A$ & Kim et al., 2013 \\
\hline & & & & $N A$ & Optical (fluorescence) & $3 \mathrm{CFU} / \mathrm{ml}$ & Jin et al., 2017 \\
\hline & & & & NA & Electrochemical & $100 \mathrm{CFU} / \mathrm{ml}$ & Wu et al., 2017 \\
\hline E10 & $\begin{array}{l}\text { Whole cell (E. coli fecal } \\
\text { isolate) }\end{array}$ & $\mathrm{NB}, 37^{\circ} \mathrm{C}$ & 0.45/E & $\begin{array}{l}\text { E. coli (non-fecal isolate), other } \\
\text { fecal isolates }\end{array}$ & $N A$ & $N A$ & Kim et al., 2013 \\
\hline $\begin{array}{l}E 1+E 2+E 10 \\
\text { (pooled) }\end{array}$ & & & & NA & Electrochemical & $371 \mathrm{CFU} / \mathrm{ml}$ & Kim et al., 2014 \\
\hline AptB12 & $\begin{array}{l}\text { Whole cell (E. coli ETEC } \\
\text { K88) }\end{array}$ & LB & E & ETEC K99, S. enteritidis, S. aureus, & Optical (fluorescence) & $1.1 \times 10^{3} \mathrm{CFU} / \mathrm{ml}$ & Peng et al., 2014 \\
\hline \multirow[t]{3}{*}{ RNAaptamer } & NR & $\mathrm{LB}, 37^{\circ} \mathrm{C}, 2-3$ & NR & NA & Electrochemical & NR & So et al., 2008 \\
\hline & & & & NA & $\begin{array}{l}\text { Immunomagnetic } \\
\text { separation and RT-PCR }\end{array}$ & $10 \mathrm{CFU} / \mathrm{ml}$ & Lee et al., 2009 \\
\hline & & & & NA & Electrochemical & 6-26 CFU/ml & Zelada-Guilleìn et al., 2010 \\
\hline
\end{tabular}


TABLE 1 | Continued

\begin{tabular}{|c|c|c|c|c|c|c|c|}
\hline Aptamer name & Target & $\begin{array}{l}\text { Culture } \\
\text { condition }^{a}\end{array}$ & $\begin{array}{l}\text { OD/Growth } \\
\text { stage }^{a, b}\end{array}$ & Counter-Selex Strains $^{c}$ & Type of sensors & LOD & References \\
\hline \multirow[t]{2}{*}{ Aptamer I-1 } & $\begin{array}{c}\text { O-antigen LPS (E. coli } \\
\text { O157:H7) }\end{array}$ & $\begin{array}{c}\text { Brucella } \\
\text { broth,37 } \mathrm{C}, 24 \mathrm{~h} \\
(+0.04 \% \\
\text { formaldehyde })\end{array}$ & NR & E. coli K12 & NA & $N A$ & Lee et al., 2012 \\
\hline & & & & NA & Electrochemical & $4 \mathrm{CFU} / \mathrm{ml}$ & Burrs et al., 2016 \\
\hline Ec3 (31) & Whole cell (E. coli DH5 2 ) & LB & 0.4 & B. subtilis & Electrochemical & $2 \times 10^{4} \mathrm{CFU} / \mathrm{ml}$ & Dua et al., 2016 \\
\hline P12-31 & Whole cell (E. coli O6) & $37^{\circ} \mathrm{C}, \mathrm{LB}$ & 0.3 & NR & $N A$ & NA & Marton et al., 2016 \\
\hline AM-6 & Whole cell (E. coli O157:H7) & LB & 0.6 & $\begin{array}{l}\text { E. coli strains O42, K12, Top10, } \\
\text { DH5 } 4 \text {, S. flexneri, S. Typhi }\end{array}$ & NA & NA & Amraee et al., 2017 \\
\hline S1 & Whole cell (E. coli O157:H7) & $\mathrm{BH}, 37^{\circ} \mathrm{C}$ & E & $\begin{array}{l}\text { S. aureus, S. Typhyimurium, } \\
\text { L. monocytogens }\end{array}$ & $\begin{array}{l}\text { Mechanical (Quartz Crystal } \\
\text { Microbalance-QCM) }\end{array}$ & $1.46 \times 10^{3} \mathrm{CFU} / \mathrm{ml}$ & Yu et al., 2018 \\
\hline Apt-5 & whole cell (E. coli O157:H7) & $\mathrm{LB}, 37^{\circ} \mathrm{C}$ & NR & E. coli ETEC and 3 other species & NA & NA & Zou et al., 2018 \\
\hline $\begin{array}{l}\text { a-aptamer/E- } \\
17 F 72^{\star}\end{array}$ & O157:H7 LPS & $\mathrm{LB}, 37^{\circ} \mathrm{C}$ & NR & NR & $N A$ & $N A$ & Bruno et al., 2009 \\
\hline $\begin{array}{l}\text { C-aptamer/E- } \\
\text { 18R72* }\end{array}$ & O157:H7 LPS & $\mathrm{LB}, 37^{\circ} \mathrm{C}$ & NR & NR & $N A$ & NA & Bruno et al., 2009 \\
\hline $\begin{array}{l}\text { a-aptamer, } \\
\text { c-aptamer }\end{array}$ & & & & $N A$ & Optical (colorimetric) & $10 \mathrm{CFU} / \mathrm{ml}$ & Wu et al., 2015 \\
\hline $\begin{array}{l}\text { a-aptamer, } \\
\text { c-aptamer }\end{array}$ & & & & NA & Optical (colorimetric) & $25 \mathrm{CFU} / \mathrm{ml}$ & Díaz-Amaya et al., 2019b \\
\hline $\begin{array}{l}\text { a-aptamer, } \\
\text { c-aptamer }\end{array}$ & & & & NA & $\begin{array}{l}\text { Optical (surface enhanced } \\
\text { raman spectroscopy-SERS) }\end{array}$ & $100 \mathrm{CFU} / \mathrm{ml}$ & Díaz-Amaya et al., 2019a \\
\hline c-aptamer & & & & $N A$ & Optical (fluorescence) & $100 \mathrm{CFU} / \mathrm{ml}$ & Hao et al., 2019 \\
\hline & & & & $N A$ & Optical (fluorescence) & $80 \mathrm{CFU} / \mathrm{ml}$ & Jiang et al., 2020 \\
\hline \multicolumn{8}{|c|}{ Helicobacter pylori } \\
\hline Hp-Ag aptamer & $\begin{array}{l}\text { Recombinant } \mathrm{Hp} \text { surface } \\
\text { antigen }\end{array}$ & NR & NR & BSA & $N A$ & NA & Gu et al., 2018 \\
\hline $\mathrm{Hp} 4$ & $\begin{array}{l}\text { Recombinant Hp surface } \\
\text { antigen }\end{array}$ & $\begin{array}{l}\text { Blood agar, } \\
37^{\circ} \mathrm{C}, 3 \text { days }\end{array}$ & NR & BSA & $N A$ & $N A$ & Yan et al., 2019 \\
\hline \multicolumn{8}{|l|}{ Legionella } \\
\hline R10C5, R10C1 & Whole cell (Lp 120292) & $\begin{array}{c}\text { CYE agar plate, } \\
37^{\circ} \mathrm{C}, 3 \text { days } \\
\text { followed by } \\
\text { AYE } \\
\text { media, } 37^{\circ} \mathrm{C}, \\
24 \mathrm{~h}\end{array}$ & 2.5/PE & $\begin{array}{c}\text { Pseudomonas putida KT2440, } \\
\text { Pseudomonas fluorescens } \\
\text { LMG1794 }\end{array}$ & $N A$ & $N A$ & Saad et al., 2020 \\
\hline \multicolumn{8}{|l|}{ NTM } \\
\hline BM2/N31 & ManLAM, M. bovis (BCG) & L-J medium & E & NR & $\begin{array}{l}\text { Optical (ELONA) } \\
\text { Electrochemical }\end{array}$ & $\begin{array}{c}10^{4} \mathrm{CFU} / \mathrm{ml} \\
\mathbf{N R}\end{array}$ & $\begin{array}{l}\text { Sun et al., } 2016 \\
\text { Sodia et al., } 2020\end{array}$ \\
\hline \multicolumn{8}{|l|}{$\begin{array}{l}\text { Pseudomonas } \\
\text { aeruginosa }\end{array}$} \\
\hline F23 & $\begin{array}{l}\text { Whole cell (P. aeruginosa } \\
\quad \text { clinical isolate) }\end{array}$ & $\begin{array}{l}\text { Mueller-Hinton } \\
(\mathrm{MH}) \text { media, } \\
37^{\circ} \mathrm{C}, 24 \mathrm{~h}\end{array}$ & NR & S. maltophilia,A. baumannii & Optical (fluorescence) & NR & Wang et al., 2011 \\
\hline
\end{tabular}


TABLE 1 | Continued

\begin{tabular}{|c|c|c|c|c|c|c|c|}
\hline Aptamer name & Target & $\begin{array}{l}\text { Culture } \\
\text { condition }^{a}\end{array}$ & $\begin{array}{l}\text { OD/Growth } \\
\text { stage }^{a, b}\end{array}$ & Counter-Selex Strains ${ }^{c}$ & Type of sensors & LOD & References \\
\hline & & & & $N A$ & $\begin{array}{l}\text { Optical (Long range } \\
\text { Surface Plasomon } \\
\text { Resonance-LSPR) }\end{array}$ & $1 \mathrm{CFU} / \mathrm{ml}$ & Hu et al., 2018 \\
\hline & & & & $N A$ & Optical (Fluorescence) & $1 \mathrm{CFU} / \mathrm{ml}$ & Zhong et al., 2018 \\
\hline & & & & $N A$ & $\begin{array}{l}\text { Electrochemical and } \\
\text { Optical (colorimetric) }\end{array}$ & $60 \mathrm{CFU} / \mathrm{ml}$ & Das et al., 2019 \\
\hline & & & & $N A$ & Electrochemical & $33 \mathrm{CFU} / \mathrm{ml}$ & Roushani et al., 2019 \\
\hline & & & & $N A$ & $\begin{array}{c}\text { Mechanical (piezoelectric } \\
\text { quartz crystal) }\end{array}$ & $9 \mathrm{CFU} / \mathrm{ml}$ & Shi et al., 2019 \\
\hline $\begin{array}{l}\text { St17Lp21, } \\
\text { St21Lp17, } \\
\text { St08Lp17 }\end{array}$ & $\begin{array}{l}\text { Biofilm-derived whole cells } \\
\text { (PA 692/ATCC 14502) }\end{array}$ & $\begin{array}{l}\text { LB broth, } 37^{\circ} \mathrm{C} \text {, } \\
16 \mathrm{~h} \text { followed } \\
\text { by } 22^{\circ} \mathrm{C}, 42 \mathrm{~h} \\
\text { to make biofilm. }\end{array}$ & E & NR & $N A$ & NA & Soundy and Day, 2017 \\
\hline $\begin{array}{l}\text { F23 + St08Lp17 } \\
\text { (pool) }\end{array}$ & & & & $N A$ & Optical (Fluorescence) & $1 \mathrm{CFU} / \mathrm{ml}$ & Zhong et al., 2020 \\
\hline \multicolumn{8}{|l|}{ Salmonella } \\
\hline \multirow[t]{6}{*}{ Aptamer 33} & OMP (S. tyhpimurium PT10) & $\begin{array}{c}\mathrm{BHI}, 37^{\circ} \mathrm{C}, 2-3 \\
\mathrm{~h}\end{array}$ & & $\begin{array}{c}\text { E. coli OMP and LPS, Salmonella } \\
\text { LPS }\end{array}$ & $\begin{array}{l}\text { Magnetic bead based pull } \\
\text { down assay and qPCR }\end{array}$ & 10-100 CFU/ml & Joshi et al., 2009 \\
\hline & & & & $N A$ & Optical (Fluorescence) & $5 \mathrm{CFU} / \mathrm{ml}$ & Duan et al., 2012 \\
\hline & & & & $N A$ & Electrochemical & $3 \mathrm{CFU} / \mathrm{ml}$ & Ma et al., 2014 \\
\hline & & & & $N A$ & Electrochemical & $55 \mathrm{CFU} / \mathrm{ml}$ & Hasan et al., 2018 \\
\hline & & & & NA & Optical (LSPR) & $30 \mathrm{CFU} / \mathrm{ml}$ & Yoo et al., 2015 \\
\hline & & & & NA & Optical (LSPR) & $10^{4} \mathrm{CFU} / \mathrm{ml}$ & Oh et al., 2017 \\
\hline \multirow[t]{3}{*}{ ST2P } & $\begin{array}{l}\text { Whole cell (S. typhimurium } \\
\text { ATCC 50761) }\end{array}$ & $\begin{array}{c}\text { BBL-BHI, } \\
37^{\circ} \mathrm{C} \text {, overnight }\end{array}$ & $0.3 / E$ & $\begin{array}{l}\text { L. monocytogenes, E. coli, S. } \\
\text { aureus, S. pneumoniae, V. } \\
\text { parahemolyticus, C. sakazakii }\end{array}$ & Optical (fluorescence) & $25 \mathrm{CFU} / \mathrm{ml}$ & Duan et al., 2013b \\
\hline & & & & NA & $\begin{array}{l}\text { Optical (Colorimetric, } \\
\text { SERS) }\end{array}$ & $10 \mathrm{CFU} / \mathrm{ml}$ & Duan et al., 2016 \\
\hline & & & & NA & Optical (Fluorescence) & $25 \mathrm{CFU} / \mathrm{ml}$ & Duan et al., 2014 \\
\hline S8-7 & $\begin{array}{l}\text { Whole cell (S. typhimurium } \\
\text { S913) }\end{array}$ & $\begin{array}{l}\text { TSB-amp, 37C, } \\
\text { overnight }\end{array}$ & NR & $\begin{array}{l}\text { L. monocytogenes Scott A E. coli } \\
\text { O157: H7, B. cereus, E. faecalis }\end{array}$ & $N A$ & $N A$ & Dwivedi et al., 2013 \\
\hline C4 & Whole cell (S. typhimurium) & $\begin{array}{l}\mathrm{BH}, 35^{\circ} \mathrm{C} \\
\text { overnight }\end{array}$ & NR & E. coli, S. enteritidis, S. aureus & NA & NA & Moon et al., 2013 \\
\hline Apt22 & Whole cell (S. paratyphi A) & NB,37C & 2.1/E & $\begin{array}{l}\text { S. Enteritidis, S. Typhimurium, S. } \\
\text { Cholerasuis, S. Arizonae }\end{array}$ & $\begin{array}{c}\text { Optical } \\
\text { (chemiluminescence) }\end{array}$ & $1000 \mathrm{CFU} / \mathrm{ml}$ & Yang et al., 2013 \\
\hline S25 & $\begin{array}{c}\text { Whole cell } \\
\text { (S. enteriditis-multiple) }\end{array}$ & TSB, overnight & NR & Salmonella serovars-multiple & & & Hyeon et al., 2012 \\
\hline SAL26 & $\begin{array}{l}\text { Whole cell (S. typhimurium } \\
\text { ATCC14028) }\end{array}$ & $\begin{array}{c}\text { TSB, } 37^{\circ} \mathrm{C} \text {, } \\
\text { overnight } \\
\text { culture followed } \\
\text { by } \mathrm{TSB}, 37^{\circ} \mathrm{C} \text {, } \\
3 \mathrm{~h} \text { then fixing } \\
\text { with methanol }\end{array}$ & E & $\begin{array}{c}4 \text { Salmonella enterica serovars and } \\
9 \text { bacterial species. }\end{array}$ & Optical (Colorimetric) & $100 \mathrm{CFU} / \mathrm{ml}$ & Lavu et al., 2016 \\
\hline
\end{tabular}


TABLE 1 | Continued

\begin{tabular}{|c|c|c|c|c|c|c|c|}
\hline Aptamer name & Target & $\begin{array}{l}\text { Culture } \\
\text { condition }^{a}\end{array}$ & $\begin{array}{l}\text { OD/Growth } \\
\text { stage }^{a, b}\end{array}$ & Counter-Selex Strains $^{c}$ & Type of sensors & LOD & References \\
\hline SAL1 & $\begin{array}{l}\text { Whole cell (S. paratyphi-A } \\
\text { ATCC 9150) }\end{array}$ & LB broth, $37^{\circ} \mathrm{C}$ & E & $\begin{array}{l}\text { S. Typhimurium, S. flexneri, E. coli } \\
\text { O157:H7, Yersinia enterocolitica }\end{array}$ & Optical (fluorescence) & $10 \mathrm{CFU} / \mathrm{ml}$ & Rm et al., 2020 \\
\hline B5 & $\begin{array}{l}\text { Whole cell (S. typhimurium } \\
\text { ATCC14028) }\end{array}$ & $\begin{array}{l}\text { BHI broth, } \\
37^{\circ} \mathrm{C}\end{array}$ & PE & $\begin{array}{c}\text { S. aureus, L. monocytogenes, } \\
\text { E. coli O157:H7 }\end{array}$ & Mechanical (QCM) & 1,000 CFU/ml & Wang L. et al., 2017 \\
\hline \multicolumn{8}{|l|}{ Shigella } \\
\hline \multirow[t]{2}{*}{ Aptamer S 1} & $\begin{array}{l}\text { Whole cell (Shigella } \\
\text { dysenteriae) }\end{array}$ & LB & E & $\begin{array}{c}\text { S. aureus, S. typhimurium, E. coli, } \\
\text { L. monocytogenes, V. } \\
\text { parahaemolyticus }\end{array}$ & Optical (Fluorescence) & $50 \mathrm{CFU} / \mathrm{ml}$ & Duan et al., 2013a \\
\hline & & & & $N A$ & Electrochemical & $1 \mathrm{CFU} / \mathrm{ml}$ & Zarei et al., 2018 \\
\hline \multirow[t]{2}{*}{ Sp1 } & $\begin{array}{l}\text { Whole cell (Shigella sonnei } \\
\text { ATCC 51334) }\end{array}$ & $\begin{array}{l}\mathrm{LB}, 37^{\circ} \mathrm{C} \\
\text { overnight }\end{array}$ & NR & $\begin{array}{l}\text { S. dysenteriae, S. flexneri, S. boydii, } \\
\text { S. typhimurium, E. coli }\end{array}$ & Optical (fluorescence) & $30 \mathrm{CFU} / \mathrm{ml}$ & Gong et al., 2015 \\
\hline & & & & NA & Optical (SERS) & $10 \mathrm{CFU} / \mathrm{ml}$ & Wu et al., 2020 \\
\hline Sp20 & $\begin{array}{l}\text { Whole cell (Shigella sonnei } \\
\text { ATCC 51334) }\end{array}$ & $\begin{array}{l}\mathrm{LB}, 37^{\circ} \mathrm{C} \\
\text { overnight }\end{array}$ & NR & $\begin{array}{l}\text { S. dysenteriae, S. flexneri, S. boydii, } \\
\text { S. typhimurium and E. coli }\end{array}$ & Optical (Fluorescence) & $30 \mathrm{CFU} / \mathrm{ml}$ & Gong et al., 2015 \\
\hline $\begin{array}{l}\text { S. flexneri } \\
\text { aptamer1 }\end{array}$ & Whole cell (Shigella flexneri) & & NR & NR & Optical (fluorescence) & $100 \mathrm{CFU} / \mathrm{ml}$ & Zhu et al., 2015 \\
\hline SS-3, SS-4 & Whole cell (Shigella sonnei) & $\mathrm{NB}, 37^{\circ} \mathrm{C}$ & NR & E. coli & Optical (Fluorescence) & $1,000 \mathrm{CFU} / \mathrm{ml}$ & Song et al., 2017 \\
\hline S. flexneri aptamer & $\begin{array}{l}\text { Whole cell (Shigella flexneri } \\
\text { ATCC 12022) }\end{array}$ & $\mathrm{NB}, 37^{\circ} \mathrm{C}, 12 \mathrm{~h}$ & NR & NR & Optical (colorimetric) & $80 \mathrm{CFU} / \mathrm{ml}$ & Feng et al., 2019 \\
\hline \multicolumn{8}{|l|}{ Vibrio cholerae } \\
\hline \multirow[t]{3}{*}{ СТ916 } & Cholerae toxin & $N A$ & $N A$ & $\begin{array}{l}\text { Ethanolamine-blocked magnetic } \\
\text { beads }\end{array}$ & Optical (colorimetric) & $2.1 \mathrm{ng} / \mathrm{ml}$ & Frohnmeyer et al., 2018 \\
\hline & & & & $N A$ & Optical (colorimetric) & $1-100 \mathrm{ng} / \mathrm{ml}$ & Frohnmeyer et al., 2019 \\
\hline & $\begin{array}{l}\text { Whole cell (V. cholerae O1 } \\
\text {-Inaba, ATCC } 39315 \text { and } \\
\text { Ogawa) }\end{array}$ & LB broth, $37^{\circ} \mathrm{C}$ & $0.4 / E$ & $\begin{array}{c}\text { E. coli O157:H7, S. a dysenteriae, } \\
\text { S. enteritidis, S. Typhimurium, } \\
\text { Yersinia spp., S. flexneri }\end{array}$ & Optical (colorimetric) & $10^{4} \mathrm{CFU} / \mathrm{ml}$ & Mojarad and Gargaria, 2020 \\
\hline \multicolumn{8}{|l|}{ Yersinia } \\
\hline N30yc5, N71yc2 & Recombinant Yop51 & NR & NR & NR & $N A$ & $N A$ & Bell et al., 1998 \\
\hline M1, M5, M7 & $\begin{array}{l}\text { Whole cell (Yersinia } \\
\text { entercolitica) }\end{array}$ & $\begin{array}{l}\text { Specific media } \\
(\mathrm{NaCl}, \text { beef } \\
\text { extract, } \\
\text { peptone, } \mathrm{pH} \\
7.2-7.4), 26^{\circ} \mathrm{C}\end{array}$ & $\begin{array}{l}0.3(\mathrm{~L}), 0.6(\mathrm{E}) \\
\quad 0.9(\mathrm{PE})\end{array}$ & $\begin{array}{c}\text { B. cereus, S. dysenteriae, L. } \\
\text { monocytogenes, S. typhimurium, } \\
\text { S. aureus, and E. coli }\end{array}$ & $N A$ & $N A$ & Shoaib et al., 2020 \\
\hline
\end{tabular}

NR, Not Reported, NA, Not applicable.

*Extrapolated from culture conditions.

a Microbial culture conditions and growth conditions are listed for aptamer development only. The Microbial culture and growth conditions used for aptasensors development are listed in Supplementary Table S1.

bState: L, lag phase; E, exponential; PE, post-exponential.

cIf number of strains used for counter selection is higher than to 5, they are listed in Supplementary Table S1. 


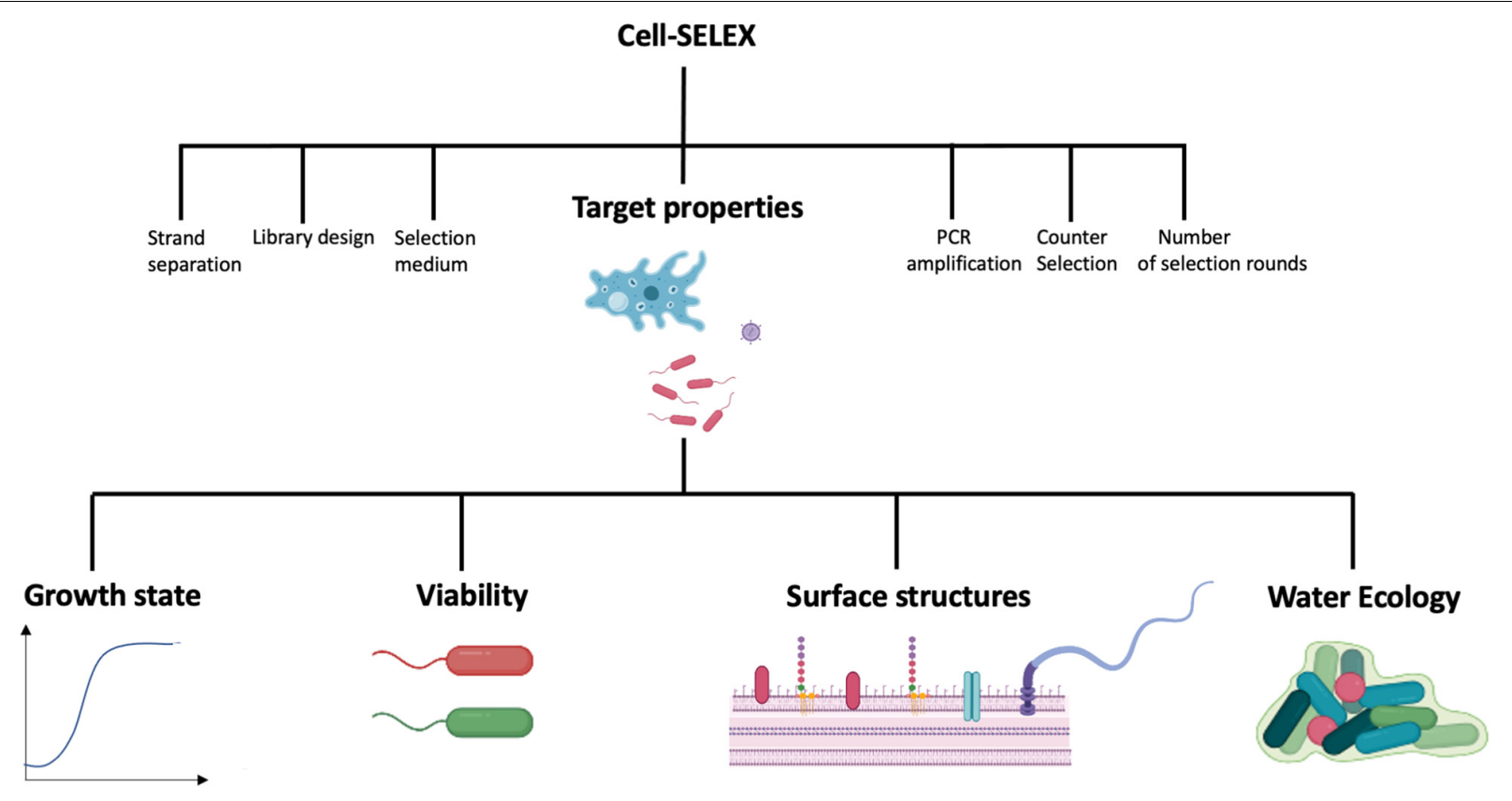

FIGURE 1 | Factors affecting Cell-SELEX and thus the efficiency of aptamers targeting water-borne microbial pathogens. Most factors have been reviewed elsewhere, except the properties of the target, which are the topics of this review. Images were created in Biorender (https://biorender.com).

of considering the target's microbial characteristics for aptamer and aptasensor development.

\section{APTAMERS TARGETING MICROBES IN SPECIFIC STATES AND GROWTH CONDITIONS}

Protozoan microbes have varying life cycles which can alternate between metabolically active feeding states, i.e., trophozoites, or inactive, dormant states such as oocysts or cysts (AguilarDíaz et al., 2011; Jain et al., 2019). Both oocysts and cysts are infectious forms that persist for long periods of time in environmental waters and resist a wide range of stressors (Omarova et al., 2018). The Cryptosporidium parvum oocystspecific aptamer R4-6 was thus developed using cell-SELEX (Table 1; Iqbal et al., 2015). A counter selection step against Giardia duodenalis, another protozoan commonly found in water samples (Ongerth, 2013; WHO, 2017) was included to enhance aptamer specificity. This aptamer was first used in multiple assay formats on electrochemical biosensing platforms to detect oocysts of $C$. parvum down to 50 oocysts in river and lake water samples (Iqbal et al., 2015; Iqbal et al., 2019). Recently, a fluorescence plate assay coupled with magnetic beads labeled with a truncated version of the aptamer R4-6, named Min_Crypto2 achieved a detection limit of 5 oocysts (Hassan et al., 2021). The low LOD of this system is promising for oocyst detection in water given that the infectious dose of C. parvum is between 10 and 30 oocysts (Jain et al., 2019). Aptamer Min_Crypto2 was selective for Cryptosporiudum species, despite differences in size amongst species, but did not bind to Giardia oocysts. These features combined with its robust performance in water samples highlights its potential for oocyst detection in water.

Bacteria suspended in water are in a different metabolic state than bacteria growing in laboratory media. For example, L. pneumophila adopts a specific regulatory program when suspended in water due to starvation ( $\mathrm{Li}$ et al., 2015). Consequently, the aptamers R10C5 and R10C1 were created by cell-SELEX using L. pneumophila suspended in water for $24 \mathrm{~h}$, to allow the bacterium to adopt the associated metabolic state (Table 1). Counter selection was performed on two Pseudomonas spp. strains, prevalent in environmental waters (Paranjape et al., 2020). Both aptamers have excellent specificity for L. pneumophila (Saad et al., 2020).

Water borne bacteria can also be biofilm-associated. These bacteria can gain adaptive traits which make it harder to eliminate or disinfect them. To that end, biofilm-derived Pseudomonas aeruginosa cells were used to select aptamers through Cell-SELEX, without counter selection (Soundy and Day, 2017). The resulting aptamers were specific for 4 out of 5 clinical Pseudomonas aeruginosa isolates, minimally labeled non-Pseudomonas bacteria, and bound to both biofilm derived and planktonic Pseudomonas cells. The authors created chimeras and generated aptamers St17Lp21, St21Lp17. The chimeric aptamers showed improved binding and enhanced specificity for Pseudomonas bacteria as compared to the parent nonchimeric aptamers but were still unable to differentiate between biofilm and planktonic cells. This is not surprising since the biofilm-derived cells were washed and vortexed to release cells and remove alginate and exopolysaccharides. Mechanical stress induced by vortexing can destroy larger surface structures such as fimbriae and flagella. The lack of counter-selection coupled 
with the vigorous washing steps may have exposed cell surface structures not unique to the biofilm-derived Pseudomonas. Using counter selection could have eliminated sequences that bind to surface structures such as LPS or ubiquitous OMPS that are common in both planktonic and biofilm-derived Pseudomonas.

Aptamers against Yersinia enterocolitica were generated using Cell-SELEX with bacteria grown at $26^{\circ} \mathrm{C}$ (Shoaib et al., 2020). After counter selecting with several bacterial pathogens, the three aptamers M1, M5, and M7 were isolated (Table 1). $Y$. enterocolitica grown at $37^{\circ} \mathrm{C}$ showed reduced binding by the aptamers compared to bacteria grown at $25^{\circ} \mathrm{C}$. Presumably this aptamer is specific for a cell surface component mostly expressed at low temperature. This study illustrates another characteristic of bacteria, which are temperature dependent surface structure and morphological changes. In the case of $Y$. enterocolitica specifically, the bacterium inhibits flagellum synthesis at $37^{\circ} \mathrm{C}$ (Kapatral et al., 1996). Components of the LPS are also temperature regulated (Białas et al., 2012).

\section{APTAMERS TARGETING VIABLE CELLS}

The ability to differentiate between dead and viable cells has important implications when assessing the risk or hazard of a microbe. For example, it would be costly and inefficient to administer shutdowns or disinfection protocols for the presence of dead pathogens in a system. The detection of viable cells is also important to determine the efficacy of water disinfection protocols. Some aptamers are able to differentiate between live and dead cells. Aptamer 33, specific for Salmonella enterica serovar Typhimurium, does not bind heat-killed cells (Table 1; Joshi et al., 2009). This aptamer might therefore be useful for monitoring the efficiency of heat-based disinfection. This aptamer is described in more detail below. Another example is aptamer ONS-23 created against whole cell C. jejuni (Table 1; Dwivedi et al., 2010). This aptamer was developed, using cellSELEX, against a chicken isolate showing characteristic C. jejuni morphology (Thomas et al., 2002). Given that C. jejuni is found on raw poultry as well as in the gastrointestinal tract and feces of animals (Mughini-Gras et al., 2016), 20 bacterial species were used for counter selection, including food-borne pathogens, enteric bacteria, non-enteric bacteria and lactic acid bacteria. ONS-23 is therefore highly specific to C. jejuni strains showing minimal binding to non-C. jejuni strains (Dwivedi et al., 2010). Furthermore,ONS-23 does not bind non-viable C. jejuni (Kim Y. J. et al., 2018) indicating that it is specific for a surface structure only present on live C. jejuni cells (Kim Y. J. et al., 2018). Though this aptamer was not tested for water application, its selective properties for viable C. jejuni makes it promising for monitoring disinfection processes.

\section{APTAMERS TARGETING SOURCE-OR APPLICATION-SPECIFIC ISOLATES}

Isolates that are representative of the sample source of the downstream application should be used during aptamer development to ensure usefulness of the aptasensor. Aptamers $\mathrm{E} 1, \mathrm{E} 2$, and E10 were generated against a non-pathogenic E. coli strain of fecal origin (Crooks strain) using cell-SELEX (Table 1; Kim et al., 2013). For counter selection a combination of fecal coliform species and two Gram positives were used. The resulting aptamers were better at binding $E$. coli isolates of fecal origin than others and showed low binding to other species including laboratory strains of E. coli (Kim et al., 2013; Jin et al., 2017; Wu et al., 2017). A detection system using aptamer E2 was able to detect the Crooks strain in spiked tap water, pond water and milk, making it promising for E. coli detection in water (Jin et al., 2017).

\section{APTAMERS TARGETING SPECIFIC SURFACE STRUCTURES}

Surface structures can be differentially expressed in response to growth states and environment (Justice et al., 2004; Van Der Woude and Bäumler, 2004; Liu et al., 2012; Fonseca and Swanson, 2014; Li et al., 2015). If the aptamer surface target is not differentially regulated then aptamers may bind cells in several conditions, including exponential and postexponential phase. Examples of these are the ST2P aptamer against whole cell $S$. enterica Typhimurium (Duan et al., 2013b, 2014, 2016) and the E. coli E2 aptamer (Kim et al., 2013; Jin et al., 2017; Wu et al., 2017). Instead of whole cells, surface structures related to virulence can also be used as aptamer targets. The pathotype EHEC (E. coli enterohemorrhagic) contains the infamous O157:H7 serotype which is strongly linked to deadly outbreaks from contaminated drinking water (Solomon et al., 2002; Ali, 2004; Saxena et al., 2015). For detecting this serotype, the specific variant of LPS can be exploited. E. coli aptamers a-aptamer and c-aptamer were created against LPS of E. coli O157:H7 (Table 1; Bruno et al., 2009). These aptamers were used in several aptasensing platforms to detect whole E. coli O157:H7 cells with great specificity, showing minimal signals with other serotypes (Wu et al., 2015; Díaz-Amaya et al., 2019a,b; Hao et al., 2019; Jiang et al., 2020). The aptamers could bind to heat-killed and formalin killed E. coli (Hao et al., 2019; Jiang et al., 2020). This is likely due to the fact that these treatments do not negatively affect the LPS (Gao et al., 2006; Chafin et al., 2013). This approach allowed for very specific aptamers to be developed; however, since the target persists after killing of cells, the aptamers are of limited use for monitoring the efficacy of disinfection programs in water. This illustrates the need for designing aptamers relevant to the downstream application.

Outer membrane proteins (OMP) of Typhimurium were used to create Aptamer 33. Counter selection was done with purified LPS of the Salmonella isolate as well as OMPs and LPS from E. coli. Aptamer 33 showed pan-serovar specificity, binding to seven different serovars of $S$. enterica in one study and four different S. enterica serovars in another study (Joshi et al., 2009; Hasan et al., 2018). The aptamer was used in a fluorescence aptasensor to detect whole Typhimurium in water samples from different sources highlighting its potential for detection in water (Duan et al., 2012). The aptamer does not bind to heat-killed 
Typhimurium which is to be expected as most OMPs are heat labile (Oh et al., 2017). The authors also observed that the aptamers could not bind $S$. enterica serovars Tennessee and Muenchen. This suggests that the aptamer may not have broad serovar specificity.

\section{DISCUSSION}

Aptamer-coupled biosensors are promising systems for the detection of pathogens in water samples but are limited in realworld applications. There are a few things to consider to improve aptamers practicality in aptasensing technology (Figure 1). Many studies do not explicitly report the growth states and conditions used during cell-SELEX or during subsequent testing of the aptamers (Table 1 and Supplementary Table 1). For example, $\mathrm{OD}_{600}$ values are meaningless without details about the growth conditions, including medium, temperature and aeration. We suggest that instead of reporting $\mathrm{OD}_{600}$, the growth phase should be determined and reported, as done by Zou et al. (2018), as this would offer insight into an aptamer's potential for specific applications. Regardless, it is important to keep the end goal in mind while developing aptamers. For example, monitoring efficiency of disinfection program will require discerning viable cells from dead cells. Aptamer ONS23 and Aptamer 33 are able to distinguish between live and dead cells (Joshi et al., 2009; Dwivedi et al., 2010; Oh et al., 2017; Kim Y. J. et al., 2018). A cell-SELEX strategy for such an application could use dead cells for counter selection. Another factor to consider is the physio-morphological state of microbes. This ensures that the microbial target possesses traits and characteristics that are representative of what's typically found in the environment that will be sampled. For example, biofilm-derived cells might be used (Soundy and Day, 2017), but care must be taken not to remove the biofilm-specific target when preparing the target for cell-SELEX. Alternatively, if the end goal is to detect pathogens in water, then bacteria suspended in water may be used as the target (Saad et al., 2020).

\section{REFERENCES}

Aguilar-Díaz, H., Carrero, J. C., Argüello-García, R., Laclette, J. P., and MoralesMontor, J. (2011). Cyst and encystment in protozoan parasites: optimal targets for new life-cycle interrupting strategies? Trends Parasitol. 27, 450-458. doi: 10.1016/j.pt.2011.06.003

Ahmed, A., Rushworth, J. V., Hirst, N. A., and Millner, P. A. (2014). Biosensors for whole-cell bacterial detection. Clin. Microbio. Rev. 27, 631-646.

Ali, S. H. (2004). A socio-ecological autopsy of the E. coli O157: H7 outbreak in Walkerton, Ontario, Canada. Soc. Sci. Med. 58, 2601-2612. doi: 10.1016/j. socscimed.2003.09.013

Amraee, M., Oloomi, M., Yavari, A., and Bouzari, S. (2017). DNA aptamer identification and characterization for E. coli O157 detection using cell based SELEX method. Anal. Biochem. 536, 6-44.

Bahari, D., Babamiri, B., Salimi, A., and Salimizand, H. (2021). Ratiometric fluorescence resonance energy transfer aptasensor for highly sensitive and selective detection of Acinetobacter baumannii bacteria in urine sample using carbon dots as optical nanoprobes. Talanta 221:121619. doi: 10.1016/j.talanta. 2020.121619

Bell, S. D., Denu, J. M., Dixon, J. E., and Ellington, A. D. (1998). RNA molecules that bind to and inhibit the active site of a tyrosine phosphatase. J. Biol. Chem. 273, 14309-14314. doi: 10.1074/jbc.273.23.14309
Lastly, it is not trivial to select appropriate strains for counter selection. This will impact aptamer affinities for targets in source environments. A possible approach is to use a cocktail of strains for the target species and a cocktail of species typically found in the same environment for counter-selection (Dwivedi et al., 2010; Kim et al., 2013). In conclusion, it is necessary to better elucidate the microbial target and the limitation of its cognate aptamer to help push microbial aptasensing platforms to market. As such a collaborative effort is needed between academics and stakeholders (governments, industry, engineers) to develop both transducer and aptamer technologies for specific microbial contaminants in the context of source water, taking into account the particularities of the microbe and its physiological state.

\section{AUTHOR CONTRIBUTIONS}

MS reviewed the literature and compiled the information reported here, and wrote the first draft of the manuscript. MS and SPF edited the manuscript. Both authors approved submission of the manuscript.

\section{FUNDING}

Work in our laboratory was supported by an NSERC Strategic Partnership Grant No. STPGP 521532 to SPF. MS was supported by a CRIPA scholarship supported by the Fonds de recherche du Queibec-Nature et technologies no. RS-170946. MS was also supported by a FRQNT doctoral scholarship.

\section{SUPPLEMENTARY MATERIAL}

The Supplementary Material for this article can be found online at: https://www.frontiersin.org/articles/10.3389/fmicb. 2021.643797/full\#supplementary-material

Białas, N., Kasperkiewicz, K., Radziejewska-Lebrecht, J., and Skurnik, M. (2012). Bacterial Cell Surface Structures in Yersinia enterocolitica. Archiv. Immunol. Ther. Exper. 60, 199-209. doi: 10.1007/s00005-012-0168-z

Bruno, J. G. (2014). Application of DNA aptamers and quantum dots to lateral flow test strips for detection of foodborne pathogens with improved sensitivity versus colloidal gold. Pathogens 3, 341-355. doi: 10.3390/pathogens3020341

Bruno, J. G., Carrillo, M. P., and Phillips, T. (2008). In vitro antibacterial effects of antilipopolysaccharide DNA aptamer-C1qrs complexes. Folia Microbiol. 53, 295-302. doi: 10.1007/s12223-008-0046-6

Bruno, J. G., Carrillo, M. P., Phillips, T., and Andrews, C. J. (2010). A novel screening method for competitive FRET-aptamers applied to E. coli assay development. J. Fluoresc. 20, 1211-1223. doi: 10.1007/s10895-010-0670-9

Bruno, J. G., Phillips, T., Carrillo, M. P., and Crowell, R. (2009). Plasticadherent DNA aptamer-magnetic bead and quantum dot sandwich assay for Campylobacter detection. J. Fluoresc. 19, 427-435. doi: 10.1007/s10895-0080429-8

Bruno, J. G., and Sivils, J. C. (2017). Further characterization and independent validation of a DNA aptamer-quantum dot-based magnetic sandwich assay for Campylobacter, Folia. Microbiology 62, 485-490. doi: 10.1007/s12223-0170520-0

Burrs, S. L., Bhargava, M., Sidhu, R., Kiernan-Lewis, J., Gomes, C., Claussen, J. C., et al. (2016). A paper based graphene-nanocauliflower hybrid composite for 
point of care biosensing. Biosens. Bioelectron. 85, 479-487. doi: 10.1016/j.bios. 2016.05.037

Cesewski, E., and Johnson, B. N. (2020). Electrochemical biosensors for pathogen detection. Biosens. Bioelectron. 2020:112214. doi: 10.1016/j.bios.2020.112214

Chafin, D., Theiss, A., Roberts, E., Borlee, G., Otter, M., and Baird, G. S. (2013). Rapid two-temperature formalin fixation. PLoS One 8:e54138. doi: 10.1371/ journal.pone.0054138

Chand, R., and Neethirajan, S. (2017). Microfluidic platform integrated with graphene-gold nano-composite aptasensor for one-step detection of norovirus. Biosens. Bioelectron. 98, 47-53. doi: 10.1016/j.bios.2017.06.026

Chen, W., Teng, J., Yao, L., Xu, J., and Liu, G. (2020). Selection of specific DNA aptamers for hetero-sandwich-based colorimetric determination of Campylobacter jejuni in food. J. Agric. Food. Chem. 68, 8455-8461. doi: 10. 1021/acs.jafc.0c02865

Crivianu-Gaita, V., and Thompson, M. (2016). Aptamers, antibody scFv, and antibody Fab'fragments: an overview and comparison of three of the most versatile biosensor biorecognition elements. Biosens. Bioelectron. 85, 32-45. doi: 10.1016/j.bios.2016.04.091

Darmostuk, M., Rimpelova, S., Gbelcova, H., and Ruml, T. (2015). Current approaches in SELEX: an update to aptamer selection technology. Biotechnol. Adv. 33, 1141-1161. doi: 10.1016/j.biotechadv.2015.02.008

Das, R., Dhiman, A., Kapil, A., Bansal, V., and Sharma, T. K. (2019). Aptamer-mediated colorimetric and electrochemical detection of Pseudomonas aeruginosa utilizing peroxidase-mimic activity of gold NanoZyme. Anal. Bioanal. Chem. 411, 1229-1238. doi: 10.1007/s00216-018-1555-z

Dehghani, Z., Hosseini, M., Mohammadnejad, J., Bakhshi, B., and Rezayan, A. H. (2018). Colorimetric aptasensor for Campylobacter jejuni cells by exploiting the peroxidase like activity of Au@Pd nanoparticles. Mikrochim. Acta 185:448.

Díaz-Amaya, S., Lin, L. K., Deering, A. J., and Stanciu, L. A. (2019a). Aptamerbased SERS biosensor for whole cell analytical detection of E. coli O157:H7. Anal. Chim. Acta 1081, 146-156. doi: 10.1016/j.aca.2019.07.028

Díaz-Amaya, S., Zhao, M., Lin, L. K., Ostos, C., Allebach, J. P., Chiu, G. T., et al. (2019b). Inkjet printed nanopatterned aptamer-based sensors for improved optical detection of foodborne pathogens. Small 15:e1805342.

Dua, P., Ren, S., Lee, S. W., Kim, J. K., Shin, H. S., Jeong, O. C., et al. (2016). CellSELEX based identification of an RNA Aptamer for Escherichia coli and its use in various detection formats. Mol. Cells 39, 807-813. doi: 10.14348/molcells. 2016.0167

Duan, N., Chang, B., Zhang, B., Wang, H. Z., and Wu, S. (2016). Salmonella typhimurium detection using a surface-enhanced Raman scattering-based aptasensor. Intern. J. Food Microbiol. 218, 38-43. doi: 10.1016/j.ijfoodmicro. 2015.11.006

Duan, N., Ding, X., Wu, S., Xia, Y., Ma, X., Wang, Z., et al. (2013a). In vitro selection of a DNA aptamer targeted against Shigella dysenteriae. J. Microbiol. Methods 94, 170-174. doi: 10.1016/j.mimet.2013.06.016

Duan, N., Wu, S., Chen, X., Huang, Y., Xia, Y., Ma, X., et al. (2013b). Selection and characterization of aptamers against Salmonella typhimurium using whole-bacterium systemic evolution of ligands by exponential enrichment (SELEX). J. Agric. Food Chem. 61, 3229-3234. doi: 10.1021/jf400 $767 \mathrm{~d}$

Duan, N., Wu, S., Ma, X., Xia, Y., and Wang, Z. (2014). A universal fluorescent aptasensor based on AccuBlue dye for the detection of pathogenic bacteria. Anal. Biochem. 454, 1-6. doi: 10.1016/j.ab.2014.03.005

Duan, N., Wu, S., Zhu, C., Ma, X., Wang, Z., Yu, Y., et al. (2012). Dual-color upconversion fluorescence and aptamer-functionalized magnetic nanoparticles-based bioassay for the simultaneous detection of Salmonella typhimurium and Staphylococcus aureus. Analyt. Chim. Acta 723, 1-6. doi: 10.1016/j.aca.2012.02.011

Dwivedi, H. P., Smiley, R. D., and Jaykus, L. A. (2010). Selection and characterization of DNA aptamers with binding selectivity to Campylobacter jejuni using whole-cell SELEX. Appl. Microbiol. Biotechnol. 87, 2323-2334. doi: 10.1007/s00253-010-2728-7

Dwivedi, H. P., Smiley, R. D., and Jaykus, L. A. (2013). Selection of DNA aptamers for capture and detection of Salmonella typhimurium using a whole-cell SELEX approach in conjunction with cell sorting. Appl. Microbiol. Biotechnol. 97, 3677-3686. doi: 10.1007/s00253-013-4766-4

Elshafey, R., Siaj, M., and Zourob, M. M. (2015). DNA aptamers selection and characterization for development of label-free impedimetric aptasensor for neurotoxin anatoxin-a. Biosens. Bioelectron. 68, 295-302. doi: 10.1016/j.bios. 2015.01.002

Escudero-Abarca, B. I., Suh, S. H., Moore, M. D., Dwivedi, H. P., and Jaykus, L. A. (2014). Selection, characterization and application of nucleic acid aptamers for the capture and detection of human norovirus strains. PLoS One 9:e106805. doi: 10.1371/journal.pone.0106805

Feng, J., Shen, Q., Wu, J., Dai, Z., and Wang, Y. (2019). Naked-eyes detection of Shigella flexneri in food samples based on a novel gold nanoparticle-based colorimetric aptasensor. Food Control 98, 333-341. doi: 10.1016/j.foodcont. 2018.11.048

Fonseca, M. V., and Swanson, M. S. (2014). Nutrient salvaging and metabolism by the intracellular pathogen Legionella pneumophila. Front. Cell. Infect. Microbiol. 4:12. doi: 10.3389/fcimb.2014.00012

Frohnmeyer, E., Frisch, F., Falke, S., Betzel, C., and Fischer, M. (2018). Highly affine and selective aptamers against cholera toxin as capture elements in magnetic bead-based sandwich ELAA. J. Biotechnol. 269, 35-42. doi: 10.1016/j.jbiotec. 2018.01.012

Frohnmeyer, E., Tuschel, N., Sitz, T., Hermann, C., Dahl, G. T., Schulz, F., et al. (2019). Aptamer lateral flow assays for rapid and sensitive detection of cholera toxin. Analyst 144, 1840-1849. doi: 10.1039/c8an01616j

Gao, B., Wang, Y., and Tsan, M.-F. (2006). The heat sensitivity of cytokineinducing effect of lipopolysaccharide. J. Leukocyte Biol. 80, 359-366. doi: 10. 1189/jlb.1205738

Gao, R., Zhong, Z., Gao, X., and Jia, L. (2018). Graphene oxide quantum dots assisted construction of fluorescent aptasensor for rapid detection of Pseudomonas aeruginosa in food samples. J. Agric. Food. Chem. 66, 1089810905. doi: 10.1021/acs.jafc.8b02164

Gargano, J. W., Adam, E. A., Collier, S. A., Fullerton, K. E., Feinman, S. J., and Beach, M. J. (2017). Mortality from selected diseases that can be transmitted by water - United States, 2003-2009. J. Water Health 15, 438-450. doi: 10.2166/ wh. 2017.301

Gentry-Shields, J., Wang, A., Cory, R. M., and Stewart, J. R. (2013). Determination of specific types and relative levels of QPCR inhibitors in environmental water samples using excitation-emission matrix spectroscopy and PARAFAC. Water Res. 47, 3467-3476. doi: 10.1016/j.watres.2013.03.049

Giamberardino, A., Labib, M., Hassan, E. M., Tetro, J. A., Springthorpe, S., Sattar, S. A., et al. (2013). Ultrasensitive norovirus detection using DNA aptasensor technology. PLoS One 8:e79087. doi: 10.1371/journal.pone.007 9087

Gong, W., Duan, N., Wu, S., Huang, Y., Chen, X., and Wang, Z. (2015). Selection, identification, and application of dual DNA aptamers against Shigella sonnei. Analyt. Methods 7, 3625-3631. doi: 10.1039/c5ay00214a

Greco, S. L., Drudge, C., Fernandes, R., Kim, J., and Copes, R. (2020). Estimates of healthcare utilisation and deaths from waterborne pathogen exposure in Ontario, Canada. Epidemiol. Infect. 148:e70.

Gu, L., Yan, W., Liu, S., Ren, W., Lyu, M., and Wang, S. (2018). Trypsin enhances aptamer screening: a novel method for targeting proteins. Anal. Biochem. 561-562, 89-95. doi: 10.1016/j.ab.2018.09.004

Hao, X., Yeh, P., Qin, Y., Jiang, Y., Qiu, Z., Li, S., et al. (2019). Aptamer surface functionalization of microfluidic devices using dendrimers as multihandled templates and its application in sensitive detections of foodborne pathogenic bacteria. Anal. Chim. Acta 1056, 96-107. doi: 10.1016/j.aca.2019. 01.035

Hasan, M. R., Pulingam, T., Appaturi, J. N., Zifruddin, A. N., Teh, S. J., Lim, T. W., et al. (2018). Carbon nanotube-based aptasensor for sensitive electrochemical detection of whole-cell Salmonella. Anal. Biochem. 554, 34-43. doi: 10.1016/j. ab.2018.06.001

Hassan, E. M., Dixon, B. R., Sattar, S. A., Stalker, A., Örmeci, B., and DeRosa, M. C. (2021). Highly sensitive magnetic-microparticle-based aptasensor for Cryptosporidium parvum oocyst detection in river water and wastewater: effect of truncation on aptamer affinity. Talanta 222:121618. doi: 10.1016/j.talanta. 2020.121618

$\mathrm{Hu}$, J., Fu, K., and Bohn, P. W. (2018). Whole-cell Pseudomonas aeruginosa localized surface plasmon resonance aptasensor. Anal. Chem. 90, 2326-2332. doi: 10.1021/acs.analchem.7b04800

Hyeon, J. Y., Chon, J. W., Choi, I. S., Park, C., Kim, D. E., and Seo, K. H. (2012). Development of RNA aptamers for detection of Salmonella Enteritidis. J. Microbiol. Methods 89, 79-82. doi: 10.1016/j.mimet.2012.01.014 
Iqbal, A., Labib, M., Muharemagic, D., Sattar, S., Dixon, B. R., and Berezovski, M. V. (2015). Detection of Cryptosporidium parvum oocysts on fresh produce using DNA aptamers. PLoS One 10:e0137455. doi: 10.1371/journal.pone.0137455

Iqbal, A., Liu, J., Dixon, B., Zargar, B., and Sattar, S. A. (2019). Development and application of DNA-aptamer-coupled magnetic beads and aptasensors for the detection of Cryptosporidium parvum oocysts in drinking and recreational water resources. Can. J. Microbiol. 65, 851-857. doi: 10.1139/cjm-2019-0153

Jain, S., Costa Melo, T. G., Dolabella, S. S., and Liu, J. (2019). Current and emerging tools for detecting protozoan cysts and oocysts in water. TrAC Trends Analyt. Chem. 121:115695. doi: 10.1016/j.trac.2019.115695

Jiang, Y., Qiu, Z., Le, T., Zou, S., and Cao, X. (2020). Developing a dual-RCA microfluidic platform for sensitive E. coli O157:H7 whole-cell detections. Anal. Chim. Acta 1127, 79-88. doi: 10.1016/j.aca.2020.06.046

Jin, B., Wang, S., Lin, M., Jin, Y., Zhang, S., Cui, X., et al. (2017). Upconversion nanoparticles based FRET aptasensor for rapid and ultrasenstive bacteria detection. Biosens. Bioelectron. 90, 525-533. doi: 10.1016/j.bios.2016.10.029

Joshi, R., Janagama, H., Dwivedi, H. P., Senthil Kumar, T. M., Jaykus, L. A., Schefers, J., et al. (2009). Selection, characterization, and application of DNA aptamers for the capture and detection of Salmonella enterica serovars. Mol. Cell. Probes. 23, 20-28. doi: 10.1016/j.mcp.2008.10.006

Justice, S. S., Hung, C., Theriot, J. A., Fletcher, D. A., Anderson, G. G., Footer, M. J., et al. (2004). Differentiation and developmental pathways of uropathogenic Escherichia coli in urinary tract pathogenesis. Proc. Natl. Acad. Sci. U.S.A. 101, 1333-1338. doi: 10.1073/pnas.0308125100

Kapatral, V., Olson, J. W., Pepe, J. C., Miller, V. L., and Minnich, S. A. (1996). Temperature-dependent regulation of Yersinia enterocolitica class III flagellar genes. Mol. Microbiol. 19, 1061-1071. doi: 10.1046/j.1365-2958.1996.452978.x

Kaur, H. (2018). Recent developments in cell-SELEX technology for aptamer selection. Biochim. Biophys. Acta Gen. Subj. 1862, 2323-2329. doi: 10.1016/j. bbagen.2018.07.029

Kim, B., Chung, K. W., and Lee, J. H. (2018). Non-stop aptasensor capable of rapidly monitoring norovirus in a sample. J. Pharm. Biomed. Anal. 152, 315-321. doi: 10.1016/j.jpba.2018.02.022

Kim, Y. J., Kim, H. S., Chon, J. W., Kim, D. H., Hyeon, J. Y., and Seo, K. H. (2018). New colorimetric aptasensor for rapid on-site detection of Campylobacter jejuni and Campylobacter coli in chicken carcass samples. Anal. Chim. Acta 1029, 78-85. doi: 10.1016/j.aca.2018.04.059

Kim, Y. S., Chung, J., Song, M. Y., Jurng, J., and Kim, B. C. (2014). Aptamer cocktails: enhancement of sensing signals compared to single use of aptamers for detection of bacteria. Biosens. Bioelectron. 54, 195-198. doi: 10.1016/j.bios. 2013.11.003

Kim, Y. S., Song, M. Y., Jurng, J., and Kim, B. C. (2013). Isolation and characterization of DNA aptamers against Escherichia coli using a bacterial cellsystematic evolution of ligands by exponential enrichment approach. Analyt. Biochem. 436, 22-28. doi: 10.1016/j.ab.2013.01.014

Kumar, N., Hu, Y., Singh, S., and Mizaikoff, B. (2018). Emerging biosensor platforms for the assessment of water-borne pathogens. Analyst 143, 359-373. doi: 10.1039/c7an00983f

Lavu, P. S., Mondal, B., Ramlal, S., Murali, H. S., and Batra, H. V. (2016). Selection and characterization of aptamers using a modified whole cell bacterium SELEX for the detection of Salmonella enterica Serovar typhimurium. ACS Comb. Sci. 18, 292-301. doi: 10.1021/acscombsci.5b00123

Lee, H.-J., Kim, B. C., Kim, K. W., Kim, Y. K., Kim, J., and Oh, M. K. (2009). A sensitive method to detect Escherichia coli based on immunomagnetic separation and real-time PCR amplification of aptamers. Biosens. Bioelectron. 24, 3550-3555. doi: 10.1016/j.bios.2009.05.010

Lee, Y. J., Han, S. R., Maeng, J. S., Cho, Y. J., and Lee, S. W. (2012). In vitro selection of Escherichia coli O157:H7-specific RNA aptamer. Biochem. Biophys. Res. Commun. 417, 414-420. doi: 10.1016/j.bbrc.2011.11.130

Li, J., Yang, S., Zuo, C., Dai, L., Guo, Y., and Xie, G. (2020). Applying CRISPRCas12a as a signal amplifier to construct biosensors for Non-DNA targets in ultralow concentrations. ACS Sens. 5, 970-977. doi: 10.1021/acssensors. 9b02305

Li, L., Mendis, N., Trigui, H., and Faucher, S. P. (2015). Transcriptomic changes of Legionella pneumophila in water. BMC Genom. 16:637. doi: 10.1186/s12864015-1869-6

Liu, B., Hu, B., Zhou, Z., Guo, D., Guo, X., Ding, P., et al. (2012). A novel nonhomologous recombination-mediated mechanism for Escherichia coli unilateral flagellar phase variation. Nucleic Acids Res. 40, 4530-4538. doi: 10.1093/nar/ gks040

Luo, C., Lei, Y., Yan, L., Yu, T., Li, Q., Zhang, D., et al. (2012). A rapid and sensitive aptamer-based electrochemical biosensor for direct detection of Escherichia coli O111. Electroanalysis 24, 1186-1191. doi: 10.1002/elan.201100700

Lv, J., Zhao, S., Wu, S., and Wang, Z. (2017). Upconversion nanoparticles grafted molybdenum disulfide nanosheets platform for microcystin-LR sensing. Biosens. Bioelectron. 90, 203-209. doi: 10.1016/j.bios.2016.09.110

Ma, X., Jiang, Y., Jia, F., Yu, Y., Chen, J., and Wang, Z. (2014). An aptamer-based electrochemical biosensor for the detection of Salmonella. J. Microbiol. Methods 98, 94-98. doi: 10.1016/j.mimet.2014.01.003

Marton, S., Cleto, F., Krieger, M. A., and Cardoso, J. (2016). Isolation of an Aptamer that binds specifically to E. coli. PLoS One 11:e0153637. doi: 10.1371/journal. pone. 0153637

McClung, R. P., Roth, D. M., Vigar, M., Roberts, V. A., Kahler, A. M., Cooley, L. A., et al. (2017). Waterborne disease outbreaks associated with environmental and undetermined exposures to water-United States, 2013-2014. Morbid. Mortal. Weekly Rep. 66:1222.

McConnell, E. M., Nguyen, J., and Li, Y. (2020). Aptamer-based biosensors for environmental monitoring. Front. Chem. 8:434. doi: 10.3389/fchem.2020.00434

McKeague, M., McConnell, E. M., Cruz-Toledo, J., Bernard, E. D., Pach, A., Mastronardi, E., et al. (2015). Analysis of in vitro aptamer selection parameters. J. Mol. Evol. 81, 150-161. doi: 10.1007/s00239-015-9708-6

Mojarad, A. E., and Gargaria, S. L. M. (2020). Aptamer-nanobody based ELASA for detection of Vibrio cholerae O1. Iran J. Microbiol. 12, 263-272.

Moon, J., Kim, G., Lee, S., and Park, S. (2013). Identification of Salmonella typhimurium-specific DNA aptamers developed using whole-cell SELEX and FACS analysis. J. Microbiol. Methods 95, 162-166. doi: 10.1016/j.mimet.2013. 08.005

Morales, M. A., and Halpern, J. M. (2018). Guide to selecting a biorecognition element for biosensors. Bioconjug. Chem. 29, 3231-3239. doi: 10.1021/acs. bioconjchem.8b00592

Mughini-Gras, L., Penny, C., Ragimbeau, C., Schets, F. M., Blaak, H., Duim, B., et al. (2016). Quantifying potential sources of surface water contamination with Campylobacter jejuni and Campylobacter coli. Water Res. 101, 36-45. doi: 10.1016/j.watres.2016.05.069

Ng, A., Chinnappan, R., Eissa, S., Liu, H., Tlili, C., and Zourob, M. (2012). Selection, characterization, and biosensing application of high affinity congener-specific microcystin-targeting aptamers. Environ. Sci. Technol. 46, 10697-10703. doi: $10.1021 / \mathrm{es} 301686 \mathrm{k}$

Oh, S. Y., Heo, N. S., Shukla, S., Cho, H. J., Vilian, A. E., Kim, J., et al. (2017). Development of gold nanoparticle-aptamer-based LSPR sensing chips for the rapid detection of Salmonella typhimurium in pork meat. Sci. Rep. 7, 1-10.

Omarova, A., Tussupova, K., Berndtsson, R., Kalishev, M., and Sharapatova, K. (2018). Protozoan parasites in drinking water: a system approach for improved water, sanitation and hygiene in developing countries. Intern. J. Environ. Res. Public Health 15:495. doi: 10.3390/ijerph15030495

Ongerth, J. E. (2013). The concentration of Cryptosporidium and Giardia in waterthe role and importance of recovery efficiency. Water Res. 47, 2479-2488. doi: 10.1016/j.watres.2013.02.015

Paranjape, K., Bédard, E., Whyte, L. G., Ronholm, J., Prévost, M., and Faucher, S. P. (2020). Presence of Legionella spp. in cooling towers: the role of microbial diversity, Pseudomonas, and continuous chlorine application. Water Res. 169:115252. doi: 10.1016/j.watres.2019.115252

Payment, P., and Locas, A. (2011). Pathogens in water: value and limits of correlation with microbial indicators. Groundwater 49, 4-11. doi: 10.1111/j. 1745-6584.2010.00710.x

Peng, Z., Ling, M., Ning, Y., and Deng, L. (2014). Rapid fluorescent detection of Escherichia coli K88 based on DNA aptamer library as direct and specific reporter combined with immuno-magnetic separation. J. Fluoresc. 24, 11591168. doi: 10.1007/s10895-014-1396- $\mathrm{x}$

Queirós, R. B., de-los-Santos-Álvarez, N., Noronha, J. P., and Sales, M. G. F. (2013). A label-free DNA aptamer-based impedance biosensor for the detection of E. coli outer membrane proteins. Sens. Actuat. B Chem. 181, 766-772. doi: 10.1016/j.snb.2013.01.062

Queirós, R. B., Gouveia, C., Fernandes, J. R. A., and Jorge, P. A. S. (2014). Evanescent wave DNA-aptamer biosensor based on long period gratings for the 
specific recognition of E. coli outer membrane proteins. Biosens. Bioelectron. 62, 227-233. doi: 10.1016/j.bios.2014.06.062

Ramírez-Castillo, F. Y., Loera-Muro, A., Jacques, M., Garneau, P., Avelar-González, F. J., Harel, J., et al. (2015). Waterborne pathogens: detection methods and challenges. Pathogens 4, 307-334. doi: 10.3390/pathogens4020307

Rasoulinejad, S., and Gargari, S. L. M. (2016). Aptamer-nanobody based ELASA for specific detection of Acinetobacter baumannii isolates. J. Biotechnol. 231, 46-54. doi: 10.1016/j.jbiotec.2016.05.024

Rm, R., Maroli, N., Achuth, J., Ponmalai, K., and Kadirvelu, K. (2020). Highly adaptable and sensitive FRET-based aptamer assay for the detection of Salmonella paratyphi A. Spectrochim. Acta A Mol. Biomol. Spectrosc. 243:118662. doi: 10.1016/j.saa.2020.118662

Roushani, M., Sarabaegi, M., and Pourahmad, F. (2019). Impedimetric aptasensor for Pseudomonas aeruginosa by using a glassy carbon electrode modified with silver nanoparticles. Mikrochim. Acta 186:725.

Saad, M., Chinerman, D., Tabrizian, M., and Faucher, S. P. (2020). Identification of two aptamers binding to Legionella pneumophila with high affinity and specificity. Sci. Rep. 10:9145.

Saxena, T., Kaushik, P., and Mohan, M. K. (2015). Prevalence of E. coli O157: H7 in water sources: an overview on associated diseases, outbreaks and detection methods. Diagn. Microbiol. Infect. Dis. 82, 249-264. doi: 10.1016/j. diagmicrobio.2015.03.015

Shi, X., Zhang, J., and He, F. (2019). A new aptamer/polyadenylated DNA interdigitated gold electrode piezoelectric sensor for rapid detection of Pseudomonas aeruginosa. Biosens. Bioelectron. 132, 224-229. doi: 10.1016/j. bios.2019.02.053

Shoaib, M., Shehzad, A., Mukama, O., Raza, H., Niazi, S., Khan, I. M., et al. (2020). Selection of potential aptamers for specific growth stage detection of Yersinia enterocolitica. RSC Adv. 10, 24743-24752. doi: 10.1039/d0ra00683a

So, H. M., Park, D. W., Jeon, E. K., Kim, Y. H., Kim, B. S., Lee, C. K., et al. (2008). Detection and titer estimation of Escherichia coli using aptamer-functionalized single-walled carbon-nanotube field-effect transistors. Small 4, 197-201. doi: $10.1002 / \mathrm{smll} .200700664$

Sodia, T., Poch, D., Honda, J. R., and Bonham, A. J. (2020). Detection of mycobacterial mannose-capped Lipoarabinomannan via Electrochemical Biosensor. FASEB J. 34:1. doi: 10.1096/fasebj.2020.34.s1.07310

Solomon, E. B., Yaron, S., and Matthews, K. R. (2002). Transmission of Escherichia coli O157: $\mathrm{H} 7$ from contaminated manure and irrigation water to lettuce plant tissue and its subsequent internalization. Appl. Environ. Microbiol. 68, 397-400. doi: 10.1128/aem.68.1.397-400.2002

Song, M. S., Sekhon, S. S., Shin, W. R., Kim, H. C., Min, J., Ahn, J. Y., et al. (2017). Detecting and discriminating Shigella sonnei using an aptamerbased fluorescent biosensor platform. Molecules 22:825. doi: 10.3390/ molecules 22050825

Song, S., Wang, L., Li, J., Fan, C., and Zhao, J. (2008). Aptamer-based biosensors. TrAC Trends Analyt. Chem. 27, 108-117.

Soundy, J., and Day, D. (2017). Selection of DNA aptamers specific for live Pseudomonas aeruginosa. PLoS One 12:e0185385. doi: 10.1371/journal.pone. 0185385

Strehlitz, B., Reinemann, C., Linkorn, S., and Stoltenburg, R. (2012). Aptamers for pharmaceuticals and their application in environmental analytics. Bioanalyt. Rev. 4, 1-30. doi: 10.1007/s12566-011-0026-1

Su, C. H., Tsai, M. H., Lin, C. Y., Ma, Y. D., Wang, C. H., Chung, Y. D., et al. (2020). Dual aptamer assay for detection of Acinetobacter baumannii on an electromagnetically-driven microfluidic platform. Biosens. Bioelectron. 159:112148. doi: 10.1016/j.bios.2020.112148

Sun, X., Pan, Q., Yuan, C., Wang, Q., Tang, X. L., Ding, K., et al. (2016). A Single ssDNA aptamer binding to mannose-capped Lipoarabinomannan of Bacillus calmette-guérin enhances immunoprotective effect against Tuberculosis. J. Am. Chem. Soc. 138, 11680-11689. doi: 10.1021/jacs.6b05357

Thomas, C., Hill, D., and Mabey, M. (2002). Culturability, injury and morphological dynamics of thermophilic Campylobacter spp. within a laboratory-based aquatic model system. J. Appl. Microbiol. 92, 433-442. doi: 10.1046/j.1365-2672.2002.01550.x

Turner, A. P. (2013). Biosensors: sense and sensibility. Chem. Soc. Rev. 42, 31843196.

Van Der Woude, M. W., and Bäumler, A. J. (2004). Phase and antigenic variation in bacteria. Clin. Microbiol. Rev. 17, 581-611. doi: 10.1128/cmr.17.3.581-611.2004
Wang, H., Bédard, E., Prévost, M., Camper, A. K., Hill, V. R., and Pruden, A. (2017). Methodological approaches for monitoring opportunistic pathogens in premise plumbing: a review. Water Res. 117, 68-86. doi: 10.1016/j.watres.2017.03.046

Wang, L., Wang, R., Chen, F., Jiang, T., Wang, H., Slavik, M., et al. (2017). QCMbased aptamer selection and detection of Salmonella typhimurium. Food Chem. 221, 776-782. doi: 10.1016/j.foodchem.2016.11.104

Wang, K. Y., Zeng, Y. L., Yang, X. Y., Li, W. B., and Lan, X. P. (2011). Utility of aptamer-fluorescence in situ hybridization for rapid detection of Pseudomonas aeruginosa. Eur. J. Clin. Microbiol. Infect. Dis. 30, 273-278. doi: 10.1007/ s10096-010-1074-0

Wang, T., Chen, C., Larcher, L. M., Barrero, R. A., and Veedu, R. N. (2019). Three decades of nucleic acid aptamer technologies: lessons learned, progress and opportunities on aptamer development. Biotechnol. Adv. 37, 28-50. doi: 10.1016/j.biotechadv.2018.11.001

Weerathunge, P., Ramanathan, R., Torok, V. A., Hodgson, K., Xu, Y., Goodacre, R., et al. (2019). Ultrasensitive colorimetric detection of murine norovirus using nanozyme aptasensor. Anal. Chem. 91, 3270-3276. doi: 10.1021/acs.analchem. $8 \mathrm{~b} 03300$

WHO, (2017). Guidelines for Drinking Water Quality. Geneva: WHO.

Wu, G., Dai, Z., Tang, X., Lin, Z., Lo, P. K., Meyyappan, M., et al. (2017). Graphene field-effect transistors for the sensitive and selective detection of Escherichia coli using pyrene-tagged DNA Aptamer. Adv. Healthc. Mater. 6:736.

Wu, J. H., Wang, C. H., Ma, Y. D., and Lee, G. B. (2018). A nitrocellulose membrane-based integrated microfluidic system for bacterial detection utilizing magnetic-composite membrane microdevices and bacteria-specific aptamers. Lab. Chip 18, 1633-1640. doi: 10.1039/c81c00251g

Wu, S., Duan, N., He, C., Yu, Q., Dai, S., and Wang, Z. (2020). Surfaceenhanced Raman spectroscopic-based aptasensor for Shigella sonnei using a dual-functional metal complex-ligated gold nanoparticles dimer. Colloids Surf. B Biointerf. 190:110940. doi: 10.1016/j.colsurfb.2020.110940

Wu, W., Zhao, S., Mao, Y., Fang, Z., Lu, X., and Zeng, L. (2015). A sensitive lateral flow biosensor for Escherichia coli O157:H7 detection based on aptamer mediated strand displacement amplification. Anal. Chim. Acta 861, 62-68. doi: 10.1016/j.aca.2014.12.041

Yan, W., Gu, L., Ren, W., Ma, X., Qin, M., Lyu, M., et al. (2019). Recognition of Helicobacter pylori by protein-targeting aptamers. Helicobacter 24:e12577. doi: 10.1111/hel.12577

Yang, M., Peng, Z., Ning, Y., Chen, Y., Zhou, Q., and Deng, L. (2013). Highly specific and cost-efficient detection of Salmonella Paratyphi A combining aptamers with single-walled carbon nanotubes. Sensors 13, 6865-6881. doi: $10.3390 / \mathrm{s} 130506865$

Yang, S., Guo, Y., Fan, J., Yang, Y., Zuo, C., Bai, S., et al. (2020). A fluorometric assay for rapid enrichment and determination of bacteria by using zirconiummetal organic frameworks as both capture surface and signal amplification tag. Mikrochim. Acta 187:188.

Yoo, S. M., Kim, D. K., and Lee, S. Y. (2015). Aptamer-functionalized localized surface plasmon resonance sensor for the multiplexed detection of different bacterial species. Talanta 132, 112-117. doi: 10.1016/j.talanta.2014.09.003

Yu, X., Chen, F., Wang, R., and Li, Y. (2018). Whole-bacterium SELEX of DNA aptamers for rapid detection of E.coli O157:H7 using a QCM sensor. J. Biotechnol. 266, 39-49. doi: 10.1016/j.jbiotec.2017.12.011

Zarei, S. S., Soleimanian-Zad, S., and Ensafi, A. A. (2018). An impedimetric aptasensor for Shigella dysenteriae using a gold nanoparticle-modified glassy carbon electrode. Mikrochim. Acta 185:538.

Zelada-Guilleìn, G. A., Bhosale, S. V., Riu, J., and Rius, F. X. (2010). Real-time potentiometric detection of bacteria in complex samples. Analyt. Chem. 82, 9254-9260. doi: 10.1021/ac101739b

Zhong, Z., Gao, R., Chen, Q., and Jia, L. (2020). Dual-aptamers labeled polydopamine-polyethyleneimine copolymer dots assisted engineering a fluorescence biosensor for sensitive detection of Pseudomonas aeruginosa in food samples. Spectrochim. Acta A Mol. Biomol. Spectrosc. 224:117417. doi: 10.1016/j.saa.2019.117417

Zhong, Z., Gao, X., Gao, R., and Jia, L. (2018). Selective capture and sensitive fluorometric determination of Pseudomonas aeruginosa by using aptamer modified magnetic nanoparticles. Mikrochim. Acta 185:377.

Zhu, Q. Y., Zhang, F. R. T., Du, Y., Zhang, X. X., Lu, J. Y., Yao, Q. F., et al. (2019). Graphene-based steganographically aptasensing system for information computing, encryption and hiding, fluorescence sensing and in vivo imaging of 
fish pathogens. ACS Appl. Mater. Interf. 11, 8904-8914. doi: 10.1021/acsami. 8 b22592

Zhu, W., Li, Z., Liu, X., Yan, X., and Deng, L. (2015). Determination of Shigella flexneri by a novel fluorescent aptasensor. Analyt. Lett. 48, 2870-2881.

Zou, Y., Duan, N., Wu, S., Shen, M., and Wang, Z. (2018). Selection, Identification, and binding mechanism studies of an ssDNA Aptamer targeted to different stages of E. coli O157:H7. J. Agric. Food Chem. 66, 5677-5682. doi: 10.1021/ acs.jafc. 8 b01006

Conflict of Interest: The authors, together with Maryam Tabrizian (McGill University, Department of Biomedical Engineering), are the inventors of aptamers R10C1 and R10C5, subject of patent applications filed in United States, patent application number US 16/850,355; and in Canada - patent application number pending at the time of revised manuscript submission. At the time of submission of the manuscript, the applications were under review.

The authors declare that the research was conducted in the absence of any commercial or financial relationships that could be construed as a potential conflict of interest.

Copyright (c) 2021 Saad and Faucher. This is an open-access article distributed under the terms of the Creative Commons Attribution License (CC BY). The use, distribution or reproduction in other forums is permitted, provided the original author(s) and the copyright owner(s) are credited and that the original publication in this journal is cited, in accordance with accepted academic practice. No use, distribution or reproduction is permitted which does not comply with these terms. 